\title{
Development and application of methods to characterize micro semi-finished products and micro components
}

\author{
Bernd Köhler*, Brigitte Clausen, and Hans-Werner Zoch \\ Leibniz Institute for Materials Engineering IWT, 28359 Bremen, Germany
}

\begin{abstract}
In the production of semi-finished products for the production of microcomponents and the components themselves, the characterization of their physical properties is of particular importance. Due to the often oligocrystalline character of these semi-finished products and components, it is necessary to use a suitable testing technique for static and dynamic investigations, as the mechanical properties are not transferable from the macroscopic point of view. In addition, the micro semi-finished products and components often show inhomogeneities induced by the manufacturing process. On the one hand, these are directly reflected in the microstructure and on the other hand they have an effect on quantities such as hardness or residual stresses, which play a decisive role in the application. Mechanical testing, conventional metallography, scanning electron microscopy (SEM), electron backscatter diffraction (EBSD), ultramicrohardness testing and X-ray residual stress analysis were used as measuring and analysis techniques suitable for the sub-millimeter range. In the following, the possibilities and limitations of two these methods are illustrated using the example of mechanical testing and EBSD. In this paper several examples for possible characterization techniques are given.

Keywords: cold forming, fatigue, micro structure
\end{abstract}

\section{Mechanical testing}

\subsection{Testing system}

The mechanical properties of the manufactured semifinished products and their post-forming behaviour in the finished product have to be analysed thoroughly. Such analysis necessitate testing systems capable of meeting specific requirements for static and cyclic testing of micro specimens. The system has to allow low forces and displacements to be set and controlled with sufficient accuracy, and provide a method of strain measurement, which takes into account the mechanical sensitivity of the specimens. Specifically with a view to dynamic testing, the machine must allow for precise control of low forces and provide an adequate displacement amplitude at maximum frequency of load cycles, whilst maintaining the preset waveform (e. g. sine wave).

\subsubsection{Testing machine}

Considering all the above mentioned requirements for static and cyclic testing of micro specimens, an electrodynamic testing machine type Instron E1000 (Fig. 1) was used to characterize the mechanical properties of thin metal sheets and rotary swagged wires. The test machine is driven by a brushless linear motor and provides a maximum load capacity of $\pm 710 \mathrm{~N}$ for static tests and $\pm 1000 \mathrm{~N}$ for cyclic tests. On the one hand, this load range proved to be sufficient for micro-samples that were strongly work-hardened by forming processes. On the other hand, the load control also allows for loads of just a few Newtons, such as those occurring with sheet thicknesses in the range of $10 \mu \mathrm{m}$.

The load was measured with load cells type Instron Dynacell calibrated to ISO 7500-1 with measuring ranges of $\pm 2 \mathrm{kN}$ and $\pm 250 \mathrm{~N}$, respectively and automatic inertia compensation.

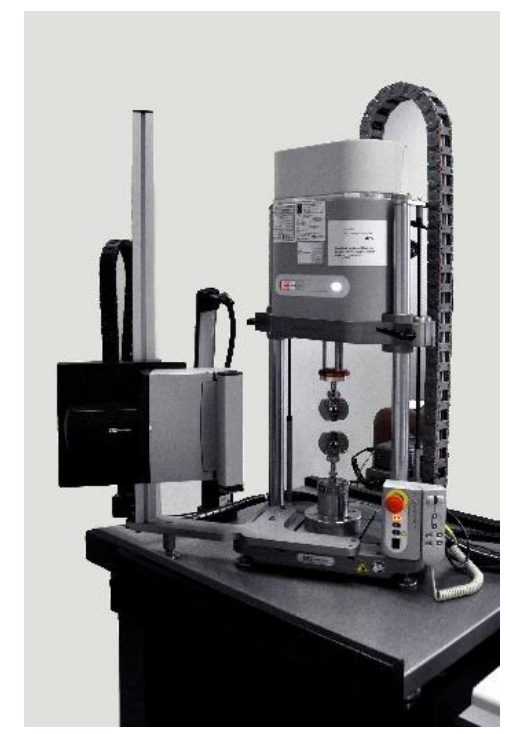

\footnotetext{
* Corresponding author: koehler@iwt-bremen.de
} 
Fig. 1. Testing machine ElectroPuls E1000.

Special attention was given to the accuracy of load control for small loads and in cyclic operation. Fig. 2 shows the measured variation of the load amplitude for a cyclic tensile test under sinusoidal load at a stress ratio $R=0.1$ and a frequency $f=20 \mathrm{~Hz}$. The specimen was a rotary swaged wire made from steel X5CrNi18-10 (AISI 304) with a diameter of $0.5 \mathrm{~mm}$. The graph shows the feedback command value of $F_{\mathrm{a}}=40.5 \mathrm{~N}$. The average load feedback amplitude determined over 2000 load cycles is $F_{\mathrm{a}}=40.487 \mathrm{~N}$ with a standard deviation of $s=0.055 \mathrm{~N}$. The deviation in the applied load is less than $1 \%$, still the first 1000 cycles were not taken into account in the calculation of the mean value, to ensure that the process of stabilization does not distort the result. Standardization of the standard deviation with respect to the measuring range of the load cell used provides:

$$
s_{\mathrm{n}}=s / 250=0.00022=0.022 \%
$$

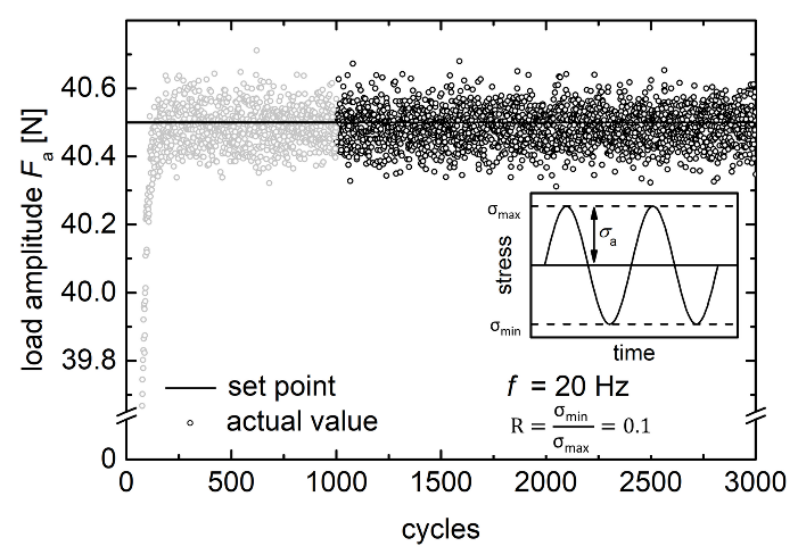

Fig. 2. Fluctuation of load amplitude during cyclic test.

The dynamic performance of the testing system is shown by way of example in Fig. 3 that illustrates the relationship between the testing frequency and the achievable displacement amplitude of the actuator for three different loading conditions. The curve plotted without a specimen installed represents a limiting curve resulting from the maximum achievable acceleration of the moving masses. Looking at the plot with installed specimen at a static mean load of $200 \mathrm{~N}$ and a load amplitude of $100 \mathrm{~N}$, it was found that a significant deviation from the limiting curve does not occur until a frequency of $100 \mathrm{~Hz}$ has been reached. When the mean load is increased to $500 \mathrm{~N}$, the curve shifts to lower frequencies, i.e. a given actuator rod amplitude will not be achievable under these conditions unless the test frequency is reduced. Basically, the performance diagram shows that testing frequencies of $100 \mathrm{~Hz}$ can be achieved with the testing system in cyclic tests, provided that the stiffness of the specimen is adequate. For the samples tested in this project, a sinusoidal loading of the samples could typically be ensured up to about $60 \mathrm{~Hz}$ during cyclic testing. At this frequency, fatigue strength tests can be carried out in a reasonable time frame. It takes about 46 hours for a run out to experience $10^{7}$ load cycles.

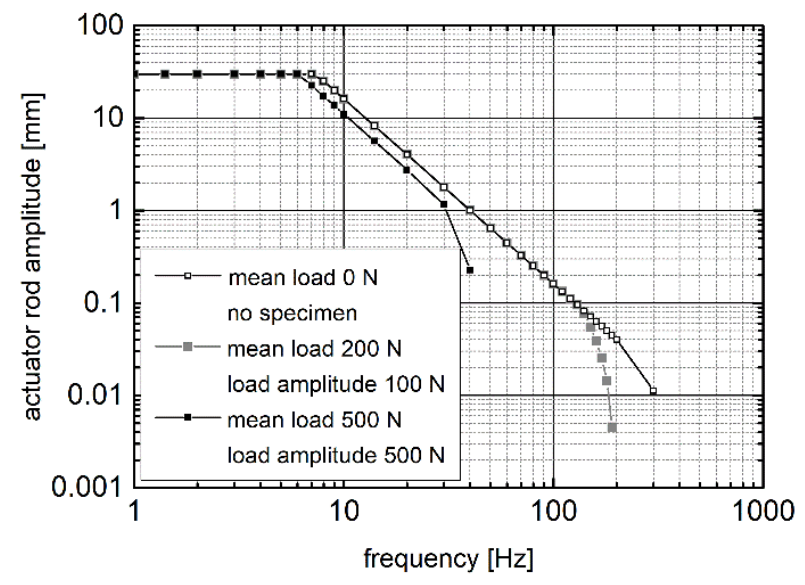

Fig. 3. Dynamic performance plot of the testing machine ElectroPuls E1000

As an example Fig. 4 shows two Woehler diagrams of wires made of steel X5CrNi18-10 (AISI 304) in the initial state as well as after cold forming by rotary swaging to a diameter of $0.5 \mathrm{~mm}$ with a feed rate of $1 \mathrm{~mm} / \mathrm{s}$. Further results are published by Köhler [8].

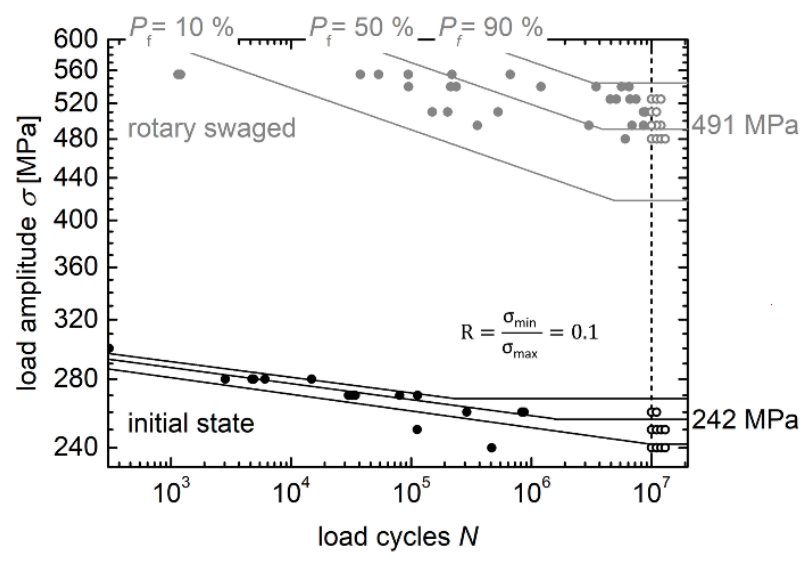

Fig. 4. Woehler diagram of $\mathrm{X} 5 \mathrm{CrNi1} 8-10$ steel wires before and after cold forming by rotary swaging $(\bullet=$ failure, $\mathbf{O}=$ run out)

The lines of the 10, 50 and $90 \%$ failure probability $P_{\mathrm{f}}$ were calculated on the basis of a Weibull distribution $[1,2]$. After rotary swaging, the fatigue strength increases from 242 to $491 \mathrm{MPa}$ and the scatter increases. A detailed discussion of the investigation can be found in Köhler and Clausen [3].

\subsubsection{Non-contact strain measurement}

In view of the micro dimensions of the test specimens (typical sheet thickness ranges between $10 \mu \mathrm{m}$ and $100 \mu \mathrm{m})$, strain measurement using specimen-contacting methods such as strain gauges or clip-on extensometers is not feasible. On the one hand, such methods involve the danger of damaging the specimens during the attachment of the respective strain measurement device, on the other 
hand, the impact of these measuring methods on the result can no longer be neglected, as it can be with larger specimens.

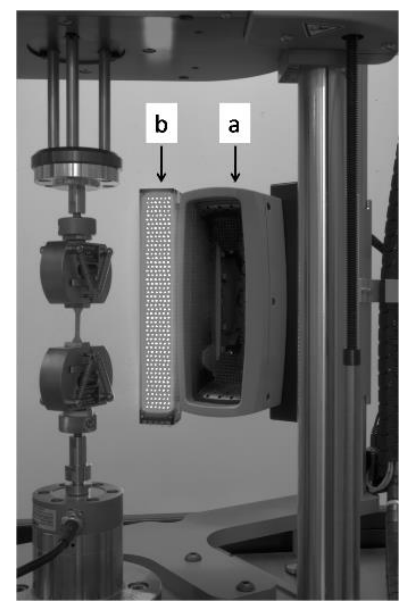

Fig. 5. Video extensometer (a) with integral illumination (b).

For this reason, a non-contacting optical strain measurement in the form of a video extensometer was chosen. The extensometer consists of a high-resolution digital video camera and a LED light source, which illuminates the specimen with pulsed, monochromatic, red polarized light (Fig. 5). In this case, strain measurement is achieved by tracing the axial movement of markings applied on the specimen with the video camera, and calculating strain by means of a real-time image processing system.

Measurement of the original gauge length, which is defined by the markings on the specimen and which is essential for strain measurement, is achieved prior to the test by the calibrated video extensometer with an absolute accuracy of $\pm 2.5 \mu \mathrm{m}$. The markings, which must ensure that they have sufficient contrast to the sample surface, take the form of two spots with a diameter between 0.5 and $3 \mathrm{~mm}$ (Fig. 6).

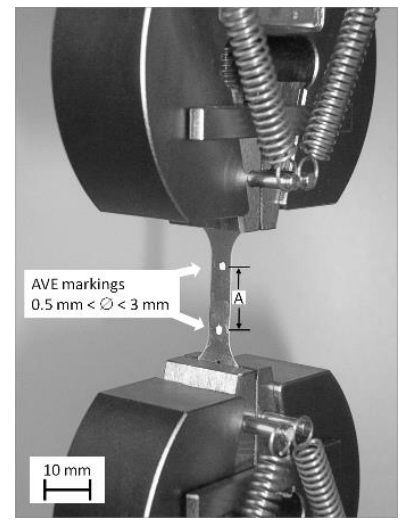

Fig. 6. Gauge marks defining the gauge length A for the AVE applied to a tensile specimen.

Fig. 7 shows the results of static tensile tests with different strain rates conducted on flat specimens. Ultimate tensile strength UTS, yield strength $\sigma_{y}$ and the elongation at fracture $A$ were determined on Al 99.5 micro sheets having a sheet thickness of $100 \mu \mathrm{m}$.

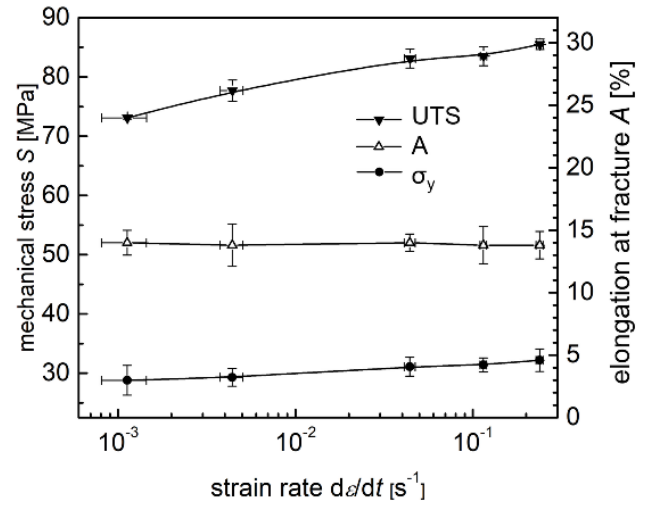

Fig. 7. Mechanical properties of a micro-flat-specimen of A199.5 (thickness $100 \mu \mathrm{m}$ ) in dependence on the strain rate.

The test system enabled the strain rate to be varied over more than two orders of magnitude between $\dot{\varepsilon}=10^{-3}$, which is approximately equivalent to that recommended in the standard DIN EN ISO $6892-1: 2016$ for tensile tests at macroscopic samples, till $\varepsilon=2 \cdot 10^{-2} \mathrm{~s}^{-1}$, the highest possible strain rate that the extensometer allows with such a specimen. This confirms that the strain rate has a significant influence on the mechanical parameters. Other than known for macro samples the ultimate tensile strength shows a clear increase with increasing strain rate. Only a weak influence is observed on the yield strength, and no influence is discernible on the elongation at break.

\subsubsection{Clamping devices}

The clamping of flat specimens is relatively easy in the standard wedge grips (Fig. 6), as long as fitted specimens can be used, so that the notching effect at the clamping device cannot have a detrimental effect. However, it is necessary to pay attention to a very exact alignment of the specimens, as otherwise wrinkles will form in the area of the clamps, which can stretch into the test area (Fig. 8). It may be advisable to use suitable auxiliary structures, such as those suggested by Hong and Hoffmann, when installing the specimens [4].

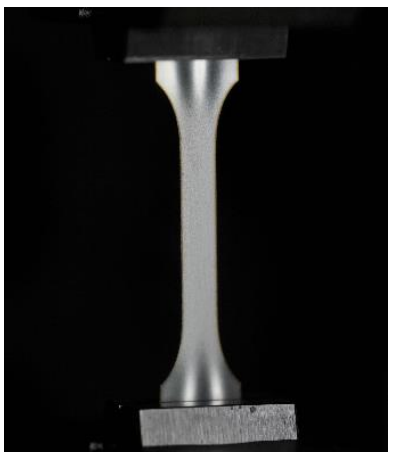

Fig. 8. Wrinkling of thin sheet tensile test specimen (thickness $100 \mu \mathrm{m})$ due to eccentric clamping in tensile direction.

The testing of round specimens, on the other hand, poses a greater challenge, as is the case, for example, with the 
cold forming process of rotary swaging, the principle of which is explained for example by Kuhfuss et al. or Piwek et al. $[5,6]$. In this process, a wire shaped semi-finished product with a diameter of $1 \mathrm{~mm}$ is rotary swaged to, for example, a diameter of $0.5 \mathrm{~mm}$ and cold worked at the same time. The mechanical characterization of the formed state involves thin round specimens, whose tension in a standard clamping device (Fig. 6) with corresponding clamping jaws (Fig. 9) always leads to the problem of the notch effect, which leads to failure at clamping in almost all samples. Thus, in the static tensile test, only the yield strength can be determined for these specimens, but neither tensile strength nor elongation at break. An evaluable cyclical testing is not possible at all.

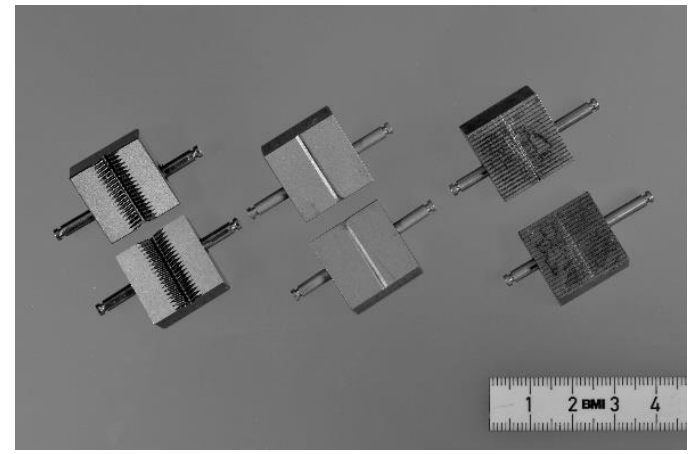

Fig. 9. Wedge jaws for use with standard clamping device (see Fig. 6) for testing round samples of different diameters.

One way of preventing failure in the clamping device is to increase the specimen cross section in the clamping area. This can be done, for example, by gluing a suitable tube onto the sample ends (Fig. 10). In principle, it is possible to ensure that the specimens break in the test area as desired and no longer in the clamping jaws. In order to obtain a sufficiently strong bond, however, it is necessary to cure the adhesive at $180{ }^{\circ} \mathrm{C}$ and thus allow the sample to undergo an unwanted heat treatment which can change the material's condition.

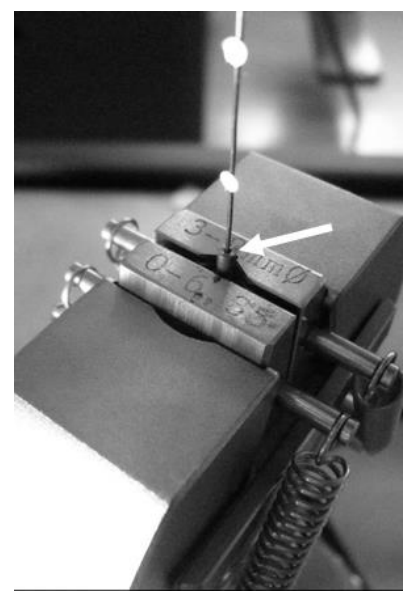

Fig. 10. Tensile test specimen of a rotary swaged wire reinforced in the clamping area with a glued-on copper tube.
In order to avoid the need for reinforcement at the clamping ends of the specimens, a concept, known from the testing of fibers, was used: The specimens are guided around a roll at both ends and only mechanically clamped at the end. In this way, the force is dissipated via the friction of the wire on the rollers and the notch effect at the end of the clamping turns out to be harmless.

Ultra-micro-hardness measurements on the rotary swaged wires made of X5CrNi18-10 showed that they undergo a strong work hardening due to the forming process and have a surface hardness up to $550 \mathrm{HV} 0.03$ [3]. In view of the fact that the clamping fixture should also be used for testing under cyclic tensile stress, it was necessary to adjust the hardness on its surface as high as possible in order to prevent the wire from working into the capstan during the test. For this reason, the nitriding steel $31 \mathrm{CrMoV} 9$ was chosen as material for the rollers. After manufacturing, the rolls were quenched and tempered $\left(870{ }^{\circ} \mathrm{C} 2 \mathrm{~h} / \mathrm{oil}+550{ }^{\circ} \mathrm{C} 2 \mathrm{~h}\right)$ and then nitrided $\left(510{ }^{\circ} \mathrm{C}\right.$ $24 \mathrm{~h})$ with characteristic number of nitrification $K_{\mathrm{n}}=3$. This way, a nitriding depth of about $0.3 \mathrm{~mm}$ could be achieved and a surface hardness of $900 \mathrm{HV} 0.1$ or 65 HRC, respectively.

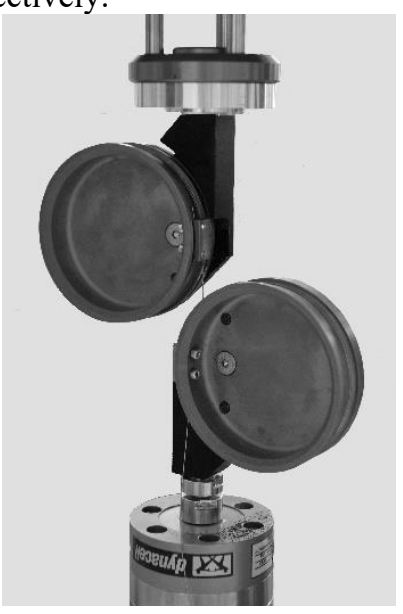

Fig. 11. Clamping device for static and cyclic testing of wires

Fig. 11 shows the manufactured clamping fixture for wires up to a diameter of $1 \mathrm{~mm}$. The specimens are guided on both sides two turns in a groove around the rollers (diameter $80 \mathrm{~mm}$ ) and are mechanically clamped at the end. In both the static and cyclic tests, failures only occurred in the test area which has a minimum length of $18 \mathrm{~mm}$. In addition, unlike the wedge clamping device, even with high-strength specimens, there are no problems with slipping during the test.

\section{Electron backscatter diffraction (EBSD)}

Electron backscatter diffraction (EBSD) was used as a further characterization method for micro cold formed semi-finished products and components. A Philips XL30 scanning electron microscope with an EBSD detector EDAX DigiView IV was available for this purpose. 


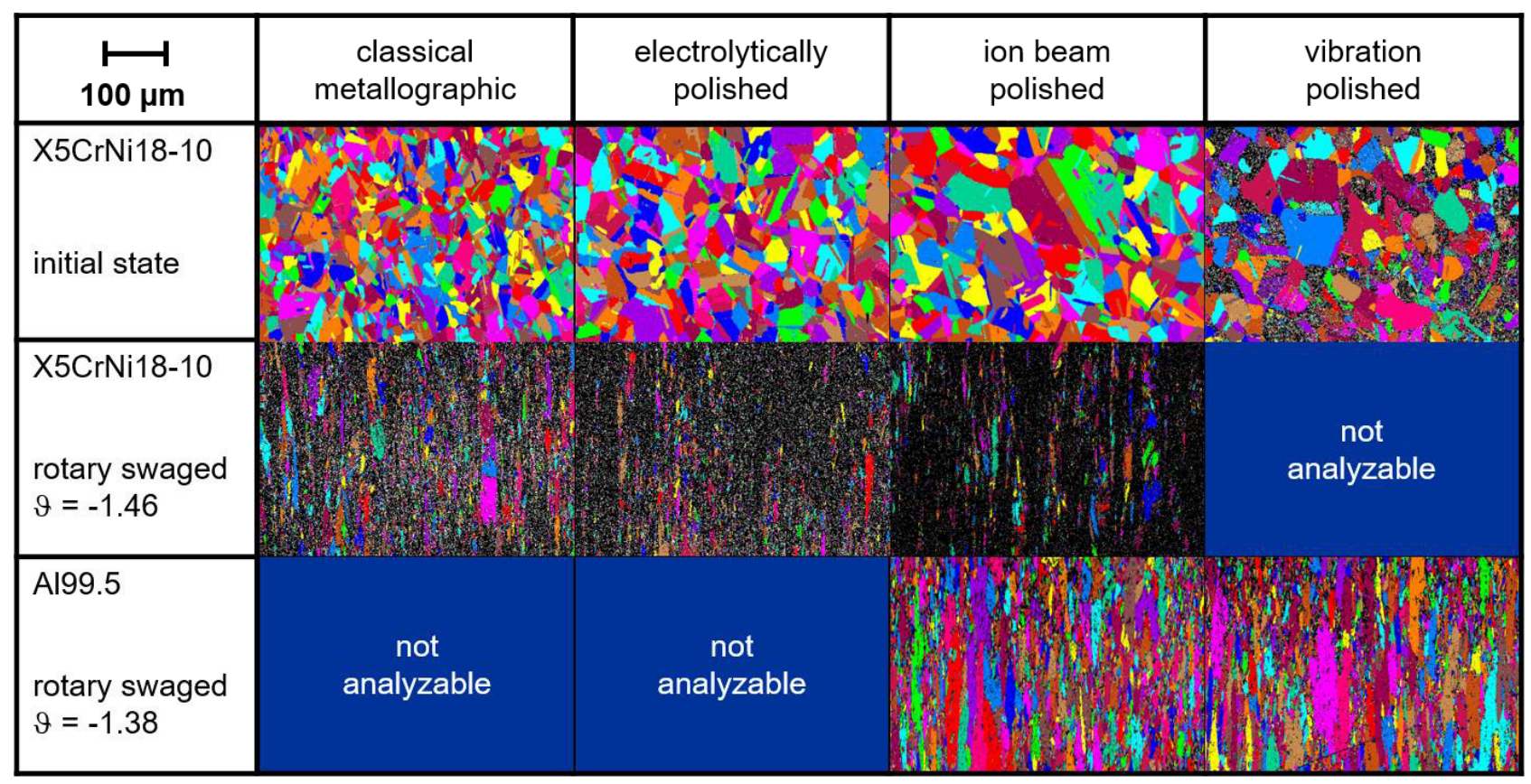

Fig. 13. Grain diagrams for comparison of different preparation methods for EBSD investigations on rotary swaged specimens and material in initial state

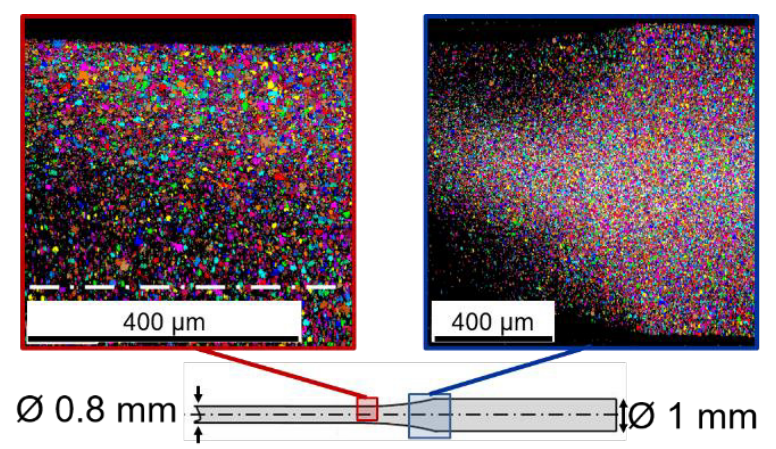

Fig. 12. EBSD grain images from the rotary swaged area (red) and the transition area (blue) of X5CrNi18-10 steel wire.

Fig. 12 shows two EBSD grain images of the centre of a cold formed wire made of steel X5CrNi18-10. The sample was rotary swaged from an initial diameter of $1 \mathrm{~mm}$ to a diameter of $0.8 \mathrm{~mm}$. This corresponds to a comparatively low degree of deformation of $\varphi=\ln \left(A / A_{0}\right)=-0.45$, where $A$ is the initial cross section and $A_{0}$ is the cross section after forming. One EBSD measurement was taken in the rotary swaged area (Fig. 12, red area), the second measurement in the transition area from the starting material to the formed area (Fig. 12, blue area).

In order to detect the poor indexability of the Kikuchi patterns in these areas, systematic investigations were carried out using various conventional preparation methods on different materials at different degrees of deformation. A selection of the results of these investigations is shown in Fig. 13: The conventional metallographic preparation, with which the sample was prepared in Fig. 12, electrolytic polishing, ion beam polishing and vibration polishing were compared on the basis of three different sample batches, steel X5CrNi18-
10 in initial state and in rotary swaged state and $\mathrm{Al}$ 99.5, also in rotary swaged condition. At $\varphi=-1.46$ and -1.38 the degree of deformation of the latter two states are significantly larger than the deformation degree of the sample from fig. 12.

It can be seen that the first three procedures lead to comparably good results when the steel is prepared in its initial state. Vibratory polishing provides in principle also large evaluable areas, but has already larger, nonindexable regions. A significantly different picture results from the corresponding strongly cold-formed steel. In this case, the vibration-polished variant could not be analyzed at all and the three other preparation methods each have very large, non indexable areas. Fig. 14 shows an enlarged view of the grain representation before (a) and after forming (b) as well as the corresponding image quality (IQ) diagrams (c and d).

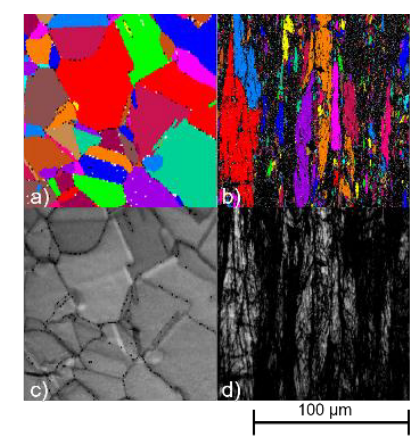

Fig. 14. Detailed depiction of the grain diagrams of X5CrNi1810 steel wires in the initial state (a) and the rotary swaged state with degree of deformation $\varphi=-1.46$ (b) and the corresponding image quality diagrams ( $\mathrm{c}$ and $\mathrm{d}$ ).

\footnotetext{
* Corresponding author: koehler@iwt-bremen.de
} 
In the latter case, the darker grey shades in the image denote lower IQ values in the evaluable areas. Any distortion to the crystal lattice within the diffracting volume produce lower quality (more diffuse) diffraction patterns. This enables the parameter to provide a qualitative description of the strain distribution in the microstructure, provided that the underlying structures can still be dissolved in principle [7]. Thus the dark, nonindexable areas are interpreted here as areas of high strain, but possibly also as very fine-grained structures. The evaluable areas of the grain diagrams show a strong grain stretching in the direction of the sample longitudinal axis compared to the initial state in the rotary swaged state. The same picture can be seen in Fig. 13 for A199.5 in the rotary swaged condition with approximately the same degree of deformation. In this case, a clear longitudinal stretching of the grains can be seen and, in contrast to steel, measurements on ion-beam and vibration-polished samples can be easily indicated. This could be an indication that the reason for the poor evaluability of the formed steel specimens is due to a fine-grained structure that lies beyond the resolution limit of the measuring system used.

In contrast to steel, the A199.5 measurements on ion-beam and vibration-polished specimens are quite easy to indicate, whereas the conventional metallographic and electrolytic preparation proves to be unsuitable. This shows that it is not possible to make a general statement about the most suitable preparation method, but that it depends on the material and its condition.

Although, for the above-mentioned reasons, only an incomplete indexing of the Kikuchi pattern is possible in the cold-formed state, at least qualitative findings can be drawn from the EBSD measurements, which cannot be obtained by conventional metallographic methods, as shown in Fig. 15. Four different evaluations of an EBSD mapping on an $\mathrm{X} 5 \mathrm{CrNi} 18-10$ steel wire in the initial state and in the rotary swaged state $(\varphi=-1.38)$ are shown. The figures show the middle plane of the specimen, with the longitudinal axis of the wires running horizontally.

In contrast to the homogeneous structure of the initial state, a strongly inhomogeneous microstructure can already be seen in the grain depiction after strong cold forming. A similar structure can be seen around the longitudinal axis of the sample as in Figure 14, consisting of relatively large and strongly stretched grains. On the other hand, the edge areas show a clearly finer structure

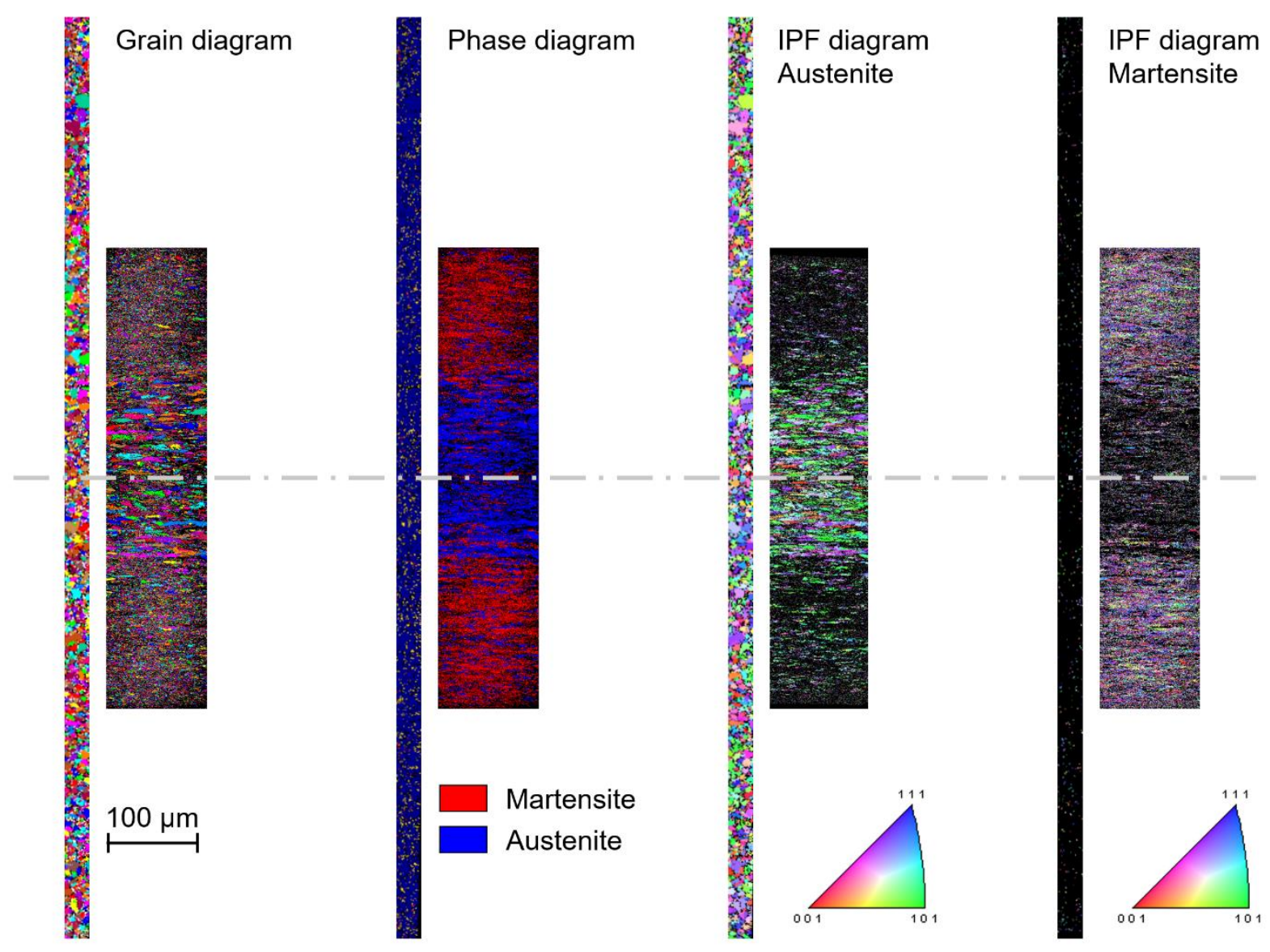

Fig. 15. EBSD mappings of X5CrNi18-10 steel wires: The figure shows a section through the specimen center plane in the initial state, $\varnothing 1 \mathrm{~mm}$ (left) and the rotary swaged state, $\varnothing 0.5 \mathrm{~mm}$ (right), $\varphi=-1.38$, in four different mappings: grain diagram, phase diagram and the inverse pole figure (IPF) diagrams of austenite respectively martensite. 
after rotary swaging, in which only very occasionally larger and stretched grains such as in the close to the axis areas can be found. In the phase representation, these peripheral areas are largely identified as martensite, whereas the central part is almost completely austenitic. Obviously, in the originally completely austenitic wire, strong deformation in the periphery areas is accompanied by formation of deformation-induced martensite, which is also confirmed by the distinct ferromagnetic properties of the material after forming.

The inverse pole figure (IPF) diagram for the austenitic phase also shows that the forming process causes texturing. The grains in the austenitic sample center preferably align themselves with the [101]-direction parallel to the sample longitudinal axis. In the predominantly martensitic peripheral regions of the sample, the IPF diagram shows a clearly purple colored portion, which can be assigned to the legend in the standard triangle according to a crystal direction between [001]- and [111]-direction, approximately in the [112]direction. In addition, there are also smaller green areas where the [101]-direction lies in the direction of the wire axis, as in the austenitic area.

Overall, the analysis using EBSD shows that the workpiece is subjected to an inhomogeneous material state due to the strong cold forming during swaging. This concerns not only the microstructure, but also other variables such as residual stress distribution across the diameter, and naturally has a decisive influence on its mechanical properties. In cyclic testing, for example, failure of the material thus formed is typically based on a very narrowly defined area within the martensitic region below the surface in which a residual stress maximum can be found [3].

\section{Summary}

The characterization of semi-finished products for the production of micro cold-formed components as well as the components themselves places special demands on the devices and methods used. Mechanical testing of such micro specimens requires testing equipment specifically adapted to their small dimensions.

The mechanical testing of such micro-samples requires a test equipment that allows the reliable measurement and control of static as well as dynamic forces in the area of a few Newtons. Tactile measurement methods, for example in strain measurement, are excluded, so that optical methods are the method of choice. Furthermore, the smaller the specimens to be tested are, the higher the demands on their handling, e. g. for precise alignment in the clamping device of the testing machine. A special challenge arises if the test specimens do not allow to be brought into a conventional sample form similar to that used in macroscopic samples. For this purpose, it may be necessary to use special devices in order to obtain valid results. This is the case, for example, in the case of semifinished products and components that have no homogeneous properties, so that any mechanical, material-removing processing for sample production would falsify the test result.
In the characterization of microstructures, conventional metallographic methods quickly reach their limits for sample sizes in the distinct sub-millimeter range. Orientation imaging microscopy, which is based on electron backscattering (EBSD), allows statements about phase distribution, grain structure and orientation, is a powerful addition. However, this method requires an extremely complex sample preparation for micro samples. In particular, it was shown that it is necessary to find out the most suitable preparation technique individually according to the material and its condition. Very strongly deformed and/or ultra-fine grain structures could only be investigated to a limited extent. However, there are at least qualitative findings to be gained which cannot be achieved with pure conventional metallography.

\section{Acknowledgments}

The authors would like to thank the German Research Foundation (DFG) for financial support of the subproject B4 "Component strength" within the collaborative research centre SFB 747 "Micro Cold Forming".

\section{References}

1. E. Macherauch, H.-W. Zoch, Praktikum in Werkstoffkunde (Vieweg + Teubner, Wiesbaden, 2011)

2. W. Weibull, Ingeniörsvetenskapsakademiens Handlingar Nr. 151 (Generalstabens Litografiska Anstalts Förlag, Stockholm, 1939)

3. B. Köhler, B. Clausen, Fortschritte in der Werkstoffprüfung für Forschung und Praxis, Proceedings Tagung Werkstoffprüfung 2017 Berlin, eds.: H. Frenz, J. B. Langer (DVM e. V., Berlin, 2017)

4. S. Hong, H. Hoffmann, Proceedings $1^{\text {st }}$ Colloquium Processscaling (BIAS Verlag, Bremen, 2003)

5. B. Kuhfuss, E. Moumi, V. Piwek, Microsystem Technologies 14, 1995 (2008)

6. V. Piwek, B. Kuhfuss, E. Moumi, M. Hork, International Journal of Material Forming 3/1, 845 (2010)

7. S. T. Wardle, L. S. Lin, A.Cetel, B. L. Adams, Proceedings $52^{\text {nd }}$ Annual Meeting of the Microscopy Society of America, eds.: G. W. Bailey, A. J. GarrettReed (San Francisco Press, San Francisco, 1994)

8. B. Köhler, B. Clausen, H.W. Zoch, Tagungsband 8. Kolloquium Mikroproduktion, 27.-28. 11. 17, Bremen, Deutschland. Vollertsen, F. Hopmann, C.; Schulze, V.; Wulfsberg, J. (Hrsg.), 91 (2017)

\footnotetext{
* Corresponding author: koehler@iwt-bremen.de
} 